\title{
Male breast cancer after heart transplantation: a Case Study from The University of California, San Francisco
}

\author{
J. L. Zakhireh ${ }^{\text {a }}$ J. W. Park ${ }^{\text {, }}$ D. McGlothlin ${ }^{\text {, }}$ L. J. Esserman ${ }^{\mathrm{a}}$ \\ ${ }^{a}$ Department of Surgery, Division of Surgical Oncology, University of California, San Francisco, USA; \\ ${ }^{b}$ Department of Madicine, Division of Hematology-Oncology, University of California, San Francisco, USA; \\ ${ }^{c}$ Department of Medicine, Division of Cardiology, University of California, San Francisco, USA.
}

\section{Presentation and diagnosis}

W. B. is a 77-year-old male with history of an orthotopic heart transplantation for non-ischemic dilated cardiomyopathy in April, 2006. He returned to a good state of health after his transplantation and was without evidence of allograft dysfunction or rejection. His immunosuppression regimen included zenapax for induction, prednisone taper, tacrolimus and cellcept, which was switched to myfortic due to gastrointestinal side effects. One year after his transplantation, he developed a left breast mass found on self-examination. Physical exam confirmed a $2.5 \times 1.5 \mathrm{~cm}^{2}$ mass just posterior and medial to the nipple-areola complex. Diagnostic mammogram and ultrasound demonstrated a $1.7 \times$ $2.4 \mathrm{~cm}^{2}$ suspicious breast mass. A core biopsy yielded grade 2 invasive ductal carcinoma, estrogen receptor positive, progesterone receptor positive and $3+$ over-expression of human epidermal growth factor HER2/neu.

A positron emission tomography with computerized tomography scan was obtained for the purpose of staging. The study again demonstrated a breast mass measuring $2.7 \times 3.3 \mathrm{~cm}^{2}$ with a standardized uptake value of 16 (Figure 1). There was no fluorodeoxyglucose (FDG) uptake in the axillary lymph nodes and no evidence of distant metastatic disease.

Correspondence to: L. J. Esserman, M.D., M.B.A., 1600 Divisadero Street, Box 1710, San Francisco, CA 94115, USA. E-mail: Laura.Esserman@ ucsfmedctr.org; Tel: 415-353-7691; Fax: 415-353-9571

Received: $21 / 08 / 08$

Accepted: 19/09/08

BCO/822/2008/CS
Other than his history of heart failure secondary to dilated cardiomyopathy, he was in good general health. Following his transplantation, his performance status was excellent for his age. He did not have a familial history of breast or ovarian cancer. His family history was only significant for heart disease.

\section{Treatment and outcome}

He underwent a left simple mastectomy with axillary sentinel lymph node biopsy, which revealed a $3.5 \mathrm{~cm}$ invasive ductal carcinoma. No lymphovascular invasion was noted. Three sentinel nodes were identified and all were negative for metastasis. The resection margins were widely clear.

His unique case presented several challenges. First, would trastuzumab be contra-indicated in a patient with a history of heart disease and heart transplantation even with normal allograft function? Second, would doxorubicin be contra-indicated as well because of the risk of heart failure? Lastly, would an antibody-based therapy be effective in a patient taking immunosuppressive medications to prevent graft rejection? Chemotherapeutic options were discussed at our multi-disciplinary conference. The recommendation was four cycles of docetaxel and cyclophosphamide with concurrent trastuzumab, followed by further trastuzumab to complete 1 year and 5 years of tamoxifen. Radiation therapy was not recommended, given his T2NO stage. $\mathrm{He}$ successfully completed the recommended chemotherapy and trastuzumab therapy without complication. 


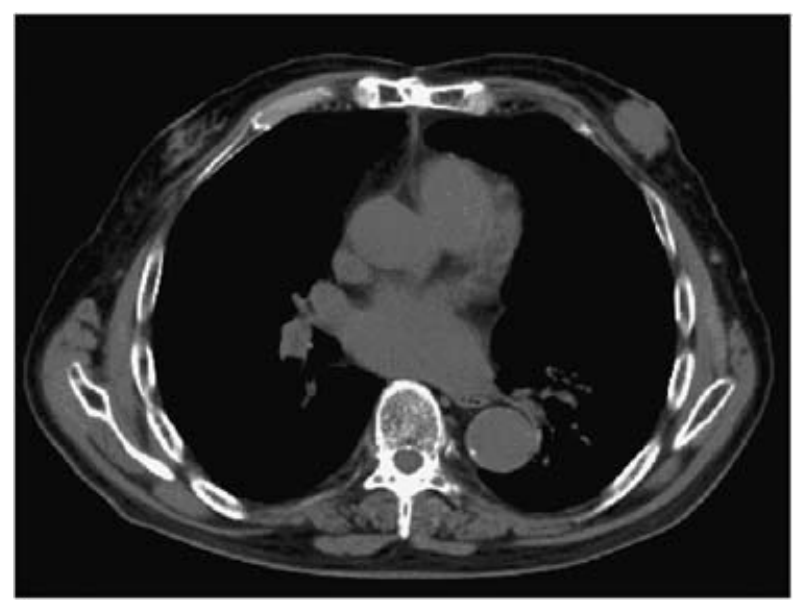

Figure 1.

This image obtained from our patient's whole-body PET/ $C T$ demonstrates the $2.7 \times 3.3 \mathrm{~cm}^{2} L$ breast mass. This mass had abnormal focal FDG avidity with a maximized standardized uptake value of 16. Neither axillary lymphadenopathy nor distant metastasis was identified.

Prior to initiation of his treatment, his immunosuppression regimen was changed to include rapamycin because of its potential anti-cancer effects. He underwent surveillance electrocardiograms and echocardiograms every 3 months while on trastuzumab and now has monthly follow-up with the heart transplant service. His last echocardiogram demonstrated normal systolic function and an ejection fraction (EF) of $65-70 \%$, which is unchanged from his EF prior to chemotherapy. $\mathrm{He}$ also undergoes routine surveillance endomyocardial biopsies, which have demonstrated normal allograft function without evidence of rejection. He is currently without evidence of recurrent breast cancer or metastatic disease. However, he has been recently diagnosed with clinical T1c prostate cancer, which was discovered following an elevated PSA value on routine testing. Given that his Gleason score was $3+3$, he has opted for active surveillance and rebiopsy.

\section{Discussion}

To our knowledge, W. B. is the first reported case of male breast cancer presenting after heart transplantation. Male breast cancer is relatively rare and represents only $1 \%$ of all diagnosed breast cancers [1]. The frequency of BRCA 1 and 2 mutations in male breast cancer patients ranges widely (4-21\%), depending on ancestry and family history [2]. W. B.'s prior probability of having a genetic mutation based on his cancer history alone was $13 \%$, as calculated using the BRCA pro model. However, his prior probability decreased to $3 \%$ when his lack of family history was accounted for in the calculation. To date, he has not undergone genetic testing.

Advances in immunosuppressive therapy have increased the chance for long-term survival in transplant recipients; however, many of these patients now face the potential complications of chronic immunosuppression, most notably malignancy and infection. The incidence of developing any malignancy after cardiac transplantation is $32 \%$ by 10 years, $21 \%$ of which are skin cancers [3]. Much less is known regarding the incidence of breast cancer in this setting. A study evaluating 25914 women receiving immunosuppression after kidney or cardiac transplantation found an overall lower than expected incidence of breast cancer (RR 0.49 for the first year post-transplant and 0.84 for subsequent years). When the smaller subset of cardiac transplant recipients were evaluated independently, the incidence was similar to expected rates in the general population [4]. Another series including 608 cardiac transplant recipients reported increased incidences of skin cancer and lung cancer, but only one case of de novo breast cancer with a mean follow-up of 4.6 years [5]. Though the data are limited, the incidence of breast and prostate cancer has not increased in transplant recipients receiving immunosuppression.

There is abundant knowledge about the biology and prognosis of female breast cancer and outcomes with chemotherapy and hormonal therapy. How this knowledge applies to men diagnosed with breast cancer is uncertain due to its rarity. Therefore, the treatment of male breast cancer is largely based upon what is extrapolated from studies on female breast cancer. Evaluation typically involves staging and risk assessment, with prognostic information gathered to help guide adjuvant treatment decisions. The overall survival for male breast cancer is similar to that of female breast cancer when matched for stage, although men often present at an older age and with more advanced disease $[6,7]$.

Our patient presented unusual challenges in the management of his primary breast cancer. One consideration was the choice of an adjuvant chemotherapy regimen. There are limited data on adjuvant chemotherapy in male breast cancer; however, a few small series have documented outcomes in male breast cancer patients with cyclophosphamide-methotrexate-5-fluoracil (CMF), 5-fluoracil-adriamycin-cyclophosphamide (FAC) and taxanes [8,9]. Due to our patient's cardiac transplant history, an anthracycline-based regimen was avoided due to its potential for cardiotoxicity. Therefore, non-anthracycline regimens, such as docetaxel and cyclophosphamide (TC), were considered. TC was 
ultimately chosen as it has been recently demonstrated to have improved 5-year disease-free survival compared to $A C$ for the adjuvant treatment of primary breast cancer in women [10].

Our patient's tumor was estrogen receptor positive, which is typical of male breast cancers (90.6\%) [6]. However, our patient's tumor also demonstrated marked over-expression of HER2/neu, which is not commonly found in male breast cancers (5\%) [11]. Based on this result and the significant positive impact on disease-free and overall survival associated with adjuvant trastuzumab therapy, the patient was treated with trastuzumab in addition to chemotherapy. Although trastuzumab therapy is also clearly associated with cardiotoxicity risk, this effect on cardiac function is frequently reversible in contrast to the fixed injury that can occur with anthracyclines [12]. Because of his excellent performance status and allograft function, the benefits of trastuzumab were favored over the potential risks. The mechanisms of action of trastuzumab include direct antiproliferative activity upon HER2overexpressing cells and indirect action via immune recognition [13]. Although the impact of ongoing immunosuppression on the second of these mechanisms is unknown, the potential benefits of this therapy were again viewed as substantial. Although, the use of trastuzumab in conjunction with TC was not specifically studied in the four major adjuvant trastuzumab trials, the established benefits of docetaxel/trastuzumab combinations in advanced breast cancer provided obvious rationale for this regimen and allowed avoidance of anthracycline- or platinum-based therapy.

Hormonal therapy represented another difficult decision. Optimal adjuvant hormonal therapy for male breast cancer is not yet defined. Tamoxifen remains a standard therapy for male breast cancer, although the use of aromatase inhibitor therapy with or without gonadal suppression has been studied in initial trials [14]. Because of the uncertainties of aromatase inhibitor-based therapy for male breast cancer and the patient's significant osteoporosis, tamoxifen was instituted following the completion of chemotherapy.

Long-term immunosuppression also poses a major clinical challenge as there are limited data supporting strategies that protect the allograft from rejection without promoting cancer. However, recent studies have found that rapamycin, a proliferation signal inhibitor used for immunosuppression, also has potent anti-cancer effects in mouse models [15-20]. Rapamycin and trastuzumab may also synergistically inhibit tumor growth in HER2/neu-positive cancer [21]. Though early outcome data in renal transplant patients are encouraging, definitive conclusions cannot be made until results from well-designed clinical trials are available. The potential that his immunosuppressive therapy may prevent the antibody-mediated activity of trastuzumab and the possible synergy between trastuzumab and rapamycin made the addition of rapamycin compelling, given the unusual nature of his situation.

In conclusion, our patient presented us with unique challenges with regard to the treatment of male breast cancer with a history of cardiac transplantation. Each patient's treatment plan should be individualized through a multi-disciplinary approach according to tumor biology, stage, allograft function and performance status.

\section{References}

1. Jemal A, Siegel R, Ward E, et al. Cancer statistics, 2008. CA Cancer J Clin 2008; 58: 71-96.

2. Liede A, Karlan BY, Narod SA. Cancer risks for male carriers of germline mutations in BRCA1 or BRCA2: a review of the literature. J Clin Oncol 2004; 22: 735-742.

3. Taylor DO, Edwards LB, Boucek MM, et al. Registry of the International Society for Heart and Lung Transplantation: twenty-fourth official adult heart transplant report - 2007. J Heart Lung Transplant 2007; 26: 769-781.

4. Stewart T, Tsai SC, Grayson H, et al. Incidence of de-novo breast cancer in women chronically immunosuppressed after organ transplantation. Lancet 1995; 346: 796-798.

5. Pham SM, Kormos RL, Landreneau RJ, et al. Solid tumors after heart transplantation: lethality of lung cancer. Ann Thorac Surg 1995; 60: 1623-1626.

6. Giordano SH, Cohen DS, Buzdar AU, et al. Breast carcinoma in men: a population-based study. Cancer 2004; 101: 51-57.

7. Giordano $\mathrm{SH}$. A review of the diagnosis and management of male breast cancer. Oncologist 2005; 10: 471-479.

8. Giordano SH, Perkins GH, Broglio K, et al. Adjuvant systemic therapy for male breast carcinoma. Cancer 2005; 104: 2359-2364.

9. Bagley CS, Wesley MN, Young RC, et al. Adjuvant chemotherapy in males with cancer of the breast. $A m \mathrm{~J}$ Clin Oncol 1987; 10: 55-60.

10. Jones SE, Savin MA, Holmes FA, et al. Phase III trial comparing doxorubicin plus cyclophosphamide with docetaxel plus cyclophosphamide as adjuvant therapy for operable breast cancer. J Clin Oncol 2006; 24: 5381-5387.

11. Muir D, Kanthan R, Kanthan SC. Male versus female breast cancers. A population-based comparative immunohistochemical analysis. Arch Pathol Lab Med 2003; 127: 36-41.

12. Ewer MS, Vooletich MT, Durand JB, et al. Reversibility of trastuzumab-related cardiotoxicity: new insights based on clinical course and response to medical treatment. J Clin Oncol 2005; 23: 7820-7826. 
13. Park JW, Neve R, Szollosi J, et al. Unraveling the biologic and clinical complexity of HER2. Clin Breast Cancer 2008; 8 (in press).

14. Nahleh ZA. Hormonal therapy for male breast cancer: a different approach for a different disease. Cancer Treat Rev 2006; 32: 101-105.

15. Gaumann A, Schlitt HJ, Geissler EK. Immunosuppression and tumor development in organ transplant recipients: the emerging dualistic role of rapamycin. Transpl Int 2008; 21: 207-217.

16. Law BK. Rapamycin: an anti-cancer immunosuppressant? Crit Rev Oncol Hematol 2005; 56: 47-60.

17. Campistol JM, Albanell J, Arns W, et al. Use of proliferation signal inhibitors in the management of post-transplant malignancies - clinical guidance. Nephrol Dial Transplant 2007; 22(Suppl 1): i36-i41.
18. Mita MM, Mita A, Rowinsky EK. The molecular target of rapamycin (mTOR) as a therapeutic target against cancer. Cancer Biol Ther 2003; 2: S169-S177.

19. Valantine $\mathrm{H}$. Is there a role for proliferation signal/mTOR inhibitors in the prevention and treatment of de novo malignancies after heart transplantation? Lessons learned from renal transplantation and oncology. $J$ Heart Lung Transplant 2007; 26: 557-564.

20. Guba M, Graeb C, Jauch KW, et al. Pro- and anti-cancer effects of immunosuppressive agents used in organ transplantation. Transplantation 2004; 77: 1777-1782.

21. Wang LH, Chan JL, Li W. Rapamycin together with herceptin significantly increased anti-tumor efficacy compared to either alone in ErbB2 over expressing breast cancer cells. Int J Cancer 2007; 121: 157-164. 